\title{
Recurrent epithelioid malignant peripheral nerve sheath tumor with neurofibromatosis type 1: A case report and literature review
}

\author{
PENG DU $^{1 *}$, JIA ZHU $^{1 *}$, ZHEN-DONG ZHANG $^{2}$, CHONG HE $^{1}$, MEI-YU YE $^{1}$, \\ YA-XIONG LIU ${ }^{1}$, QIU-HONG TIAN ${ }^{3}$ and JIN-SHENG ZENG ${ }^{1}$ \\ Departments of ${ }^{1}$ General Surgery, ${ }^{2}$ Pathology and ${ }^{3}$ Oncology, \\ The First Affiliated Hospital of Nanchang University, Nanchang, Jiangxi 330006, P.R. China
}

Received November 23, 2018; Accepted April 26, 2019

DOI: $10.3892 / \mathrm{ol} .2019 .10676$

\begin{abstract}
Malignant peripheral nerve sheath tumors (MPNSTs) are unusual and aggressive malignant soft-tissue tumors that comprise $5-10 \%$ of all soft-tissue sarcomas. Approximately $50 \%$ of MPNST cases are associated with neurofibromatosis type-1 (NF-1). As a rare MPNST subset, the epithelioid variant of MPNST (eMPNST) is histologically characterized by the predominant presence of epithelioid tumor cells, and accounts for $<5 \%$ of all MPNSTs. In addition, eMPNST is rarely associated with NF-1 when compared with conventional MPNST. Although extensive clinicopathological studies have been conducted on eMPNST, clinicians face difficulty when attempting to make an accurate diagnosis. Subsequently, the biological consequences, including recurrence, metastasis and mortality rate in patients with eMPNST remain unclear. The current study presents the case of a 71-year-old woman with eMPNST and a family history of NF-1 in whom tumors had recurred twice on the lower back. A literature search for eMPNSTs was conducted by browsing PubMed and MEDLINE for English-language articles, as well as references from review articles, and revealed 129 published cases. Only 5 cases of eMPNST were associated with NF-1. The studies were retrospectively reviewed and the clinicopathological data of the patients, including tumor site, treatment,
\end{abstract}

Correspondence to: Dr Jin-Sheng Zeng, Department of General Surgery, The First Affiliated Hospital of Nanchang University, 461 Bayi Avenue, Nanchang, Jiangxi 330006, P.R. China

E-mail: zengjinsheng0606@163.com

Dr Qiu-Hong Tian, Department of Oncology, The First Affiliated Hospital of Nanchang University, 461 Bayi Avenue, Nanchang, Jiangxi 330006, P.R. China

E-mail: eganzhou@163.com

${ }^{*}$ Contributed equally

Key words: neurofibromatosis type 1, epithelioid malignant peripheral nerve sheath tumor, recurrent, differential diagnosis, prognosis follow-up, prognosis, and immunohistochemical positivity were collected.

\section{Introduction}

Neurofibromatosis type-1 (NF-1) is a common, autosomal dominant neurocutaneous disorder with an incidence of 1:3,000 worldwide, which primarily involves the skin, bone and nervous system (1). The standard clinical diagnostic criteria for NF-1 have existed since 1987 , with $\geq 2$ criteria required for a positive diagnosis. The criteria are summarized as follows: $\geq 6$ 'café-au-lait' spots, size $>5 \mathrm{~mm}$ in prepubertal children or $>15 \mathrm{~mm}$ in post pubertal individuals, axillary or inguinal freckling, $\geq 2$ cutaneous neurofibromas, $\geq 1$ plexiform neurofibroma, $\geq 2$ Lisch nodules, a characteristic bone lesion (sphenoid wing dysplasia, dysplasia of the long bones), optic nerve glioma and a first degree relative with $\mathrm{NF}-1 \quad(1,2)$. Patients with NF-1 have an $8-13 \%$ risk of developing malignant peripheral nerve sheath tumors (MPNSTs) $(1,2)$. MPNSTs are unusual and aggressive malignant soft-tissue tumors comprising $5-10 \%$ of all soft-tissue sarcomas, and $~ 50 \%$ of all MPNST cases are associated with NF-1 (3). In addition, MPNST can also develop sporadically or be associated with exposure to radiation $(3,4)$. It has been reported that there is no sex or ethnicity predilection for MPNST (4). MPNST usually develops in the deep soft tissues and tends to appear in the extremities, trunk and the head or neck region; however, it can also rarely occur in superficial locations, including the skin or subcutaneous tissues $(5,6)$. Histologically, MPNSTs are categorized into the most common conventional type plus three specific subtypes, including rhabdomyoblastic, glandular and epithelioid tumors (7). As a subset of MPNSTs, the epithelioid variant of MPNST (eMPNST) is histologically characterized by the predominance ( $\geq 50 \%$ ) of epithelioid tumor cells and diffuse positivity for S-100 proteins; however, eMPNST is rare and accounts for $<5 \%$ of all MPNSTs (8). Furthermore, the association between eMPNST and NF-1 compared with conventional MPNST is extremely rare and occurs in $<2 \%$ of all MPNSTs (9). Although extensive clinicopathological studies have been conducted on eMPNST, it is often difficult to provide an accurate diagnosis, since its histological characteristics are similar to that of poorly differentiated cancer, malignant melanoma and other soft-tissue tumors (8). In 
addition, the uncommon association of eMPNST with NF-1 increases the difficultly to obtain a differential diagnosis. However, eMPNST displays consistent, uniform, strong and diffuse positive staining for S-100 (10). Staining for second-line melanoma markers (including HMB45, melan A, tyrosinase, and MITF) and vascular markers is usually negative (8). Therefore, immunohistochemical examination is required to diagnose eMPNST and rule out other possibilities (8). Ki-67 has a well characterized expression pattern and is known to be associated with cell proliferation (11). The classifications of neuroendocrine tumors include the Ki-67 index, high proliferation rates indicate poor prognosis and rapid tumor growth (11). MPNST has a high probability rate of local recurrence and distant metastasis, with a poor prognosis. A local recurrence rate of $40-65 \%$ and a 5-year survival rate of $23-69 \%$ have been reported $(3,12)$. However, biological complications, including recurrence, metastasis or mortality rate in patients with eMPNST remain unclear due to the small group of patients involved, the lack of large-scale clinical research and the variation in survival rates. In addition, the majority of the published literature primarily focuses on the clinicopathological characteristics of patients (13-15). The present study describes the case of a 71-year-old woman with a family history of NF-1 who was diagnosed with eMPNST and suffered twice from recurrence, with tumors located on the lower back. The present study also includes a review of the literature focusing on the clinicopathological differential diagnosis and prognosis of eMPNST.

\section{Case report}

In November 2016, a 71-year-old woman was admitted to the Department of General Surgery of The First Affiliated Hospital of Nanchang University (Nanchang, China) with an enlarged and painful mass on the lower back, which had been surgical excised twice in October 2014 and September 2015 at the Jiangxi Provincial People's Hospital (Nanchang, China). The slides and details for the specimens from October 2014 and September 2015 were available and confirmed the diagnosis of an MPNST. The mass recurred at the same site following each excision within a follow-up period of 6 months. The patient noticed the third mass at 5 months after the second operation and noted that it was gradually enlarging. Furthermore, the patient was from a family with a history of NF-1, and the records indicated that 12 people over three generations, including the patient's grandmother and mother, had suffered from NF-1. Among the family members, 4 suffered from NF-1 that developed during adolescence. The patients' older brother died at the age of 68 from other diseases, whereas the other members of the family remained alive. The diagnosis of all patients was confirmed by medical examinations (Fig. 1). There was no familial history of other major diseases. Physical examinations of the present patient revealed multiple nodules of different sizes throughout the entire body combined with numerous 'café-au-lait' spots. There was also a hard, slightly erythematous, irregular, highly swollen mass, with local surface ulceration and suppuration on the lower back, which measured 20x13x8 cm in size (Fig. 2). Computed tomography (CT) of the chest, abdomen and pelvis revealed a mass with a maximum diameter of $18 \mathrm{~cm}$, characterized as a heterogeneously dense soft-tissue mass with an irregularity border on the lower back. According to the contrast-enhanced CT scans, there was mild to moderate non-homogeneous enhancement, and no sign of distant metastases was observed (Fig. 3A). Magnetic resonance imaging (MRI) revealed that the mass had low signal intensity on T1-weighted images (Fig. 3B) and high signal intensity on T2-weighted images (Fig. 3C). Additional laboratory investigations did not reveal any other abnormalities, other than a low level of hemoglobin at $91 \mathrm{~g} / 1$ (normal range, 115-150 g/l). Comprehensive analysis of the imaging examination and the medical history of the patient were indicative of the presence of a recurrent malignant tumor on the back. During surgery, the physicians decided to perform an en bloc resection of the tumor with a negative surgical margin. Gross examination of the mass surface revealed a gray-white, fleshy tumor weighing $\sim 1,470 \mathrm{~g}$ and containing solid and cystic regions. Histologically, tumor tissue was fixed with $10 \%$ neutral formalin for $24 \mathrm{~h}$ at room temperature and embedded in paraffin. After being cut into slices ( $4 \mu \mathrm{m}$ thick) using a microtome (BQ-318D; Bona Medical Technology, Hubei, China). The paraffin sections were dewaxed by washing with xylene three times, 5 min each time, and hydrated in 100, 95, 85 and $75 \%$ ethanol solutions for $3 \mathrm{~min}$ in each. These slices were incubated in hematoxylin solution for $5 \mathrm{~min}$ at room temperature and rinsed with running water. Subsequently, the slices were immersed in eosin solution for $3 \mathrm{~min}$ at room temperature and washed again with running water. After dehydration, the slices were mounted with neutral resin and viewed under a light microscope (CX22; Olympus Corporation, Tokyo, Japan) for pathological analysis (magnification, x100 and x400). The tumor was primarily composed of cells with an epithelioid morphology arranged in a nesting and diffuse growth pattern, containing a small proportion of interspersed spindle cell components (Fig. 4A). The polygonal or round-shaped epithelioid cells had large, round, vesicular nuclei with basophilic nucleoli and abundant eosinophilic cytoplasm, and 10 mitotic figures per 10 high-power fields (HPF) (Fig. 4B). The morphology of the cells from these sections was similar to that observed in the cells from the previous surgical excision at the Jiangxi Provincial People's Hospital. The edge of the resection margin of the tumor was negative. Immunohistochemical analysis was performed on the 4- $\mu$ m-thick formalin-fixed paraffin-embedded tissue sections. Tissue sections were deparaffinized in xylene, and hydrated in graded ethanol solutions. Endogenous peroxidase activity was blocked by incubating the sections in $3 \%$ hydrogen peroxide at room temperature for $10 \mathrm{~min}$. Antigen retrieval was performed using boiling sodium citrate buffer $(\mathrm{pH}$ 6.0) in a microwave for $15 \mathrm{~min}$. Nonspecific binding was blocked in $5 \%$ bovine serum albumin (BSA) in PBS for $60 \mathrm{~min}$ at room temperature. The primary antibodies used in the lesions were, anti-S100 (cat. no. Z0311; 1:600), vimentin (cat. no. V9; 1:400), cluster of differentiation (CD) 34 (cat. no. Qbend10; 1:50), cytokeratin (cat. no. MNF116; 1:100), smooth muscle actin (SMA) (cat. no. $1 \mathrm{~A} 4 ; 1: 400$ ), melan-A (cat. no. A103; 1:50), HMB45 (cat. no. HMB45; 1:100), melanocyte inducing transcription factor (MITF) (cat. no. D5; 1:100), myogenin (cat. no. F5D; 1:50), epithelial membrane antigen (EMA) (cat. no. E29; 1:100), neurofilament (cat. no. 2F11; 1:100), desmin (cat. no. D33; 1:100) and Ki-67 (cat. no. MIB-1; 1:100) all from Dako; Agilent Technologies GmbH (Waldbronn, Germany). The 


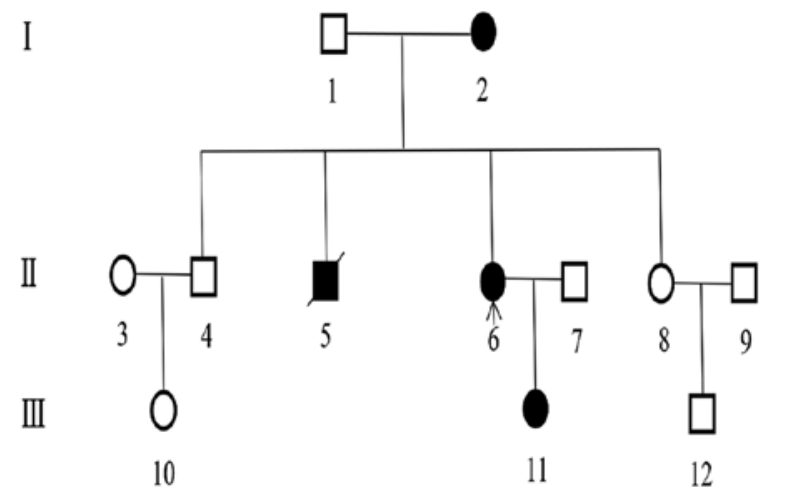

$\square$ Healthy men Diseased women OHealthy women $\boldsymbol{\varpi D e a d ~ m e n ~} \uparrow$ Precursor

Figure 1. Family tree of neurofibromatosis type 1.

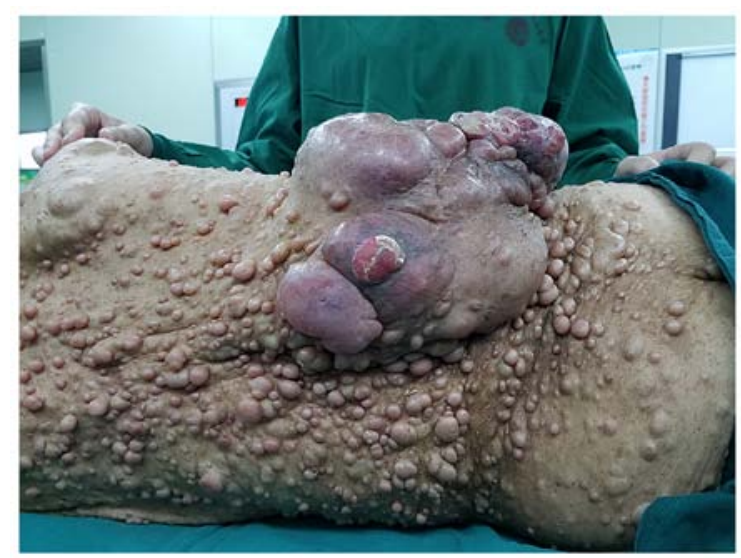

Figure 2. 'Café-au-lait' spots and nodules over the patient's entire body, with a hard, slightly erythematous, irregularly shaped, elevated tumor with local surface ulceration measuring $20 \times 13 \times 8 \mathrm{~cm}$ located on the back.

samples were incubated with the primary antibodies overnight at $4^{\circ} \mathrm{C}$ in bovine serum albumin. Subsequently the samples were incubated with a horseradish peroxidase-conjugated goat anti-mouse immunoglobulin G secondary antibody (cat. no. K5007; 1:1,000; Dako; Agileant Technologies, GmbH) for $30 \mathrm{~min}$ at $37^{\circ} \mathrm{C}$. The 3,3-diaminobenzidine chromogenic liquid was added to the sections and incubated for $10 \mathrm{~min}$ at room temperature followed by stopping with water. After restaining with hematoxylin, the sections were dehydrated using a series of ethanol solutions $(75,85,95,100$ and $100 \%$ for $3 \mathrm{~min}$ in each), and treated with xylene. The sections were sealed with neutral gum and observed using a light microscope (magnification, $\mathrm{x} 200$ ). The fraction of proliferating cells (positive for Ki-67) was calculated based on a count of at least 500 tumor cells in the relatively dense concentration of positive cancer nuclei (hot spot). The percentage of positive cells were calculated and used as the $\mathrm{Ki}-67$ index. Immunohistochemical staining revealed that tumor cells exhibited strong and diffuse positive staining for S-100 (Fig. 4C) and vimentin (Fig. 4D), whereas CD34, cytokeratin, SMA, melan-A, HMB45, MITF, myogenin, EMA, neurofilament and desmin were negative. The Ki-67 labeling index was $40 \%$ (Fig. 5). The final pathological diagnosis was eMPNST arising in the peripheral nerves. The patient recovered uneventfully following the

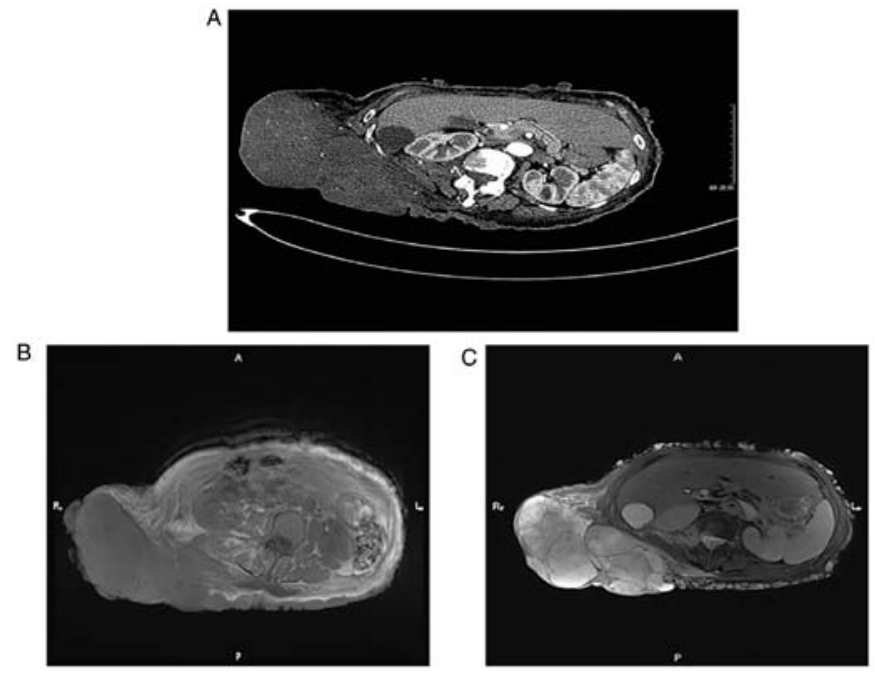

Figure 3. Preoperative computed tomography and magnetic resonance imaging scan (A). Contrast-enhanced computed tomography imaging revealed a large soft-tissue mass with mild to moderate non-homogenous enhancement measuring $18 \mathrm{~cm}$ in maximum diameter in the lower back region. Magnetic resonance imaging showed that the mass had (B) a low-intensity signal on T1-weighted images and (C) a high-intensity signal on T2-weighted images.
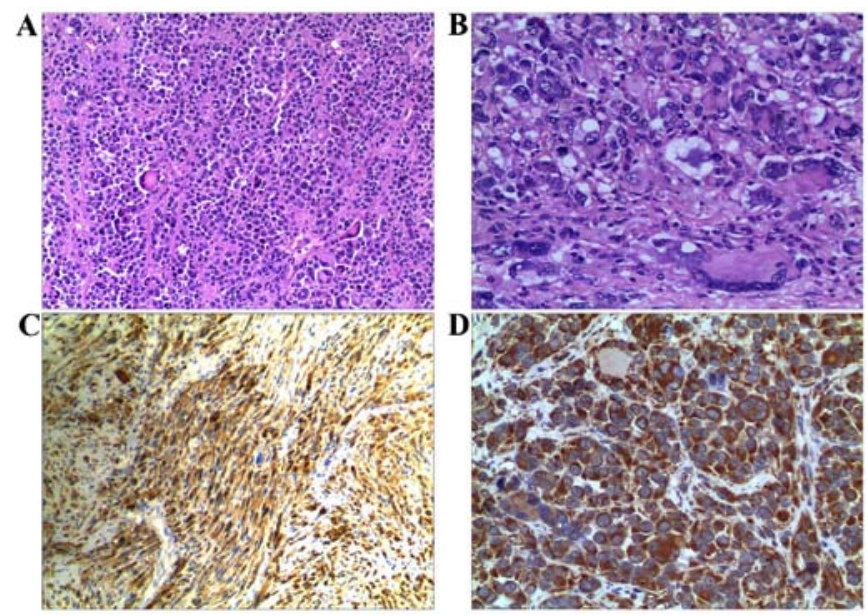

Figure 4. Microscopic findings and results of immunohistochemistry. (A) The tumor was mainly composed of cells of epithelioid morphology arranged in nesting and diffuse growth patterns, admixed with a diverse proportion of spindle cell components (H\&E staining; magnification, $\mathrm{x} 200$ ). (B) Polygonal or round-shaped epithelioid cells with large, round, vesicular nuclei, with basophilic nucleoli, and an abundant eosinophilic cytoplasm. Notable cytological atypia and mitotic figures were readily identified $(\mathrm{H} \& \mathrm{E}$ staining magnification, $\mathrm{x} 400$ ). Immunohistochemical staining demonstrated that tumor cells were diffusely positive for (C) S-100 and (D) vimentin. $\mathrm{H} \& \mathrm{E}$, hematoxylin and eosin.

operation and was subsequently referred to the Department of Oncology for further treatment due to the multiple recurrences and highly invasive nature of the tumor. A course of adjuvant radiotherapy, with a dose of 60 Gray in 30 fractions, was administered over a period of 6 weeks. At the end of all treatments, examinations for potential recurrence or metastases were performed, including CT of the chest and abdomen, a whole-body bone scan and a brain MRI, and the results were normal. Postoperative follow-up at 2 years revealed no evidence of recurrence and metastatic disease, or residual side effects from therapy. Despite the repeated relapses, metastasis 
Table I. Immunophenotypic features used for the differential diagnosis of tumors with epithelioid morphology.

\begin{tabular}{|c|c|c|c|c|c|}
\hline Protein & $\begin{array}{l}\text { Malignant } \\
\text { melanoma }\end{array}$ & $\begin{array}{l}\text { Poorly differentiated } \\
\text { carcinoma }\end{array}$ & $\begin{array}{l}\text { Epithelioid } \\
\text { sarcoma }\end{array}$ & $\begin{array}{l}\text { Myoepithelial } \\
\text { carcinoma }\end{array}$ & eMPNST \\
\hline S-100 & + & - & - & + & $+($ strong, diffuse $)$ \\
\hline Vimentin & + & - & + & + & $+($ strong, diffuse $)$ \\
\hline HMB45 & + & - & - & - & - \\
\hline Melan A & + & - & - & - & - \\
\hline SMA & - & - & \pm & \pm & - \\
\hline Desmin & - & - & \pm & \pm & - \\
\hline CD34 & - & - & \pm & - & - \\
\hline CK (AE1/AE3) & - & + & + & + & $+/-$ \\
\hline EMA & - & + & + & + & $+/-$ \\
\hline SMARCB1/INI-1 & Retained & Retained & Loss $(\sim 90 \%)$ & Loss $(\sim 40 \%)$ & Loss $(50-67 \%)$ \\
\hline
\end{tabular}

+ , positivity; \pm , occasional positivity; +/-, rare positivity. CD, cluster of differentiation; CK, cytokeratin; EMA, epithelial membrane antigen; eMPNST, epithelioid malignant peripheral nerve sheath tumor; HMB45, melanoma-associated antigen; SMA, smooth muscle actin; INI-1, nuclear integrase interactor 1 .

Table II. Immunophenotypic features used to distinguish an eMPNST from other divergent differentiations of MPNST.

\begin{tabular}{lcccc}
\hline Protein & Conventional MPNST & Glandular MPNST & MTT & eMPNST \\
\hline S-100 & + (focal) & + & + & $+($ strong, diffuse) \\
Vimentin & + & + & + & + \\
Desmin & - & - & + & $+($ strong, diffuse) \\
MyoD1 & - & - & - & - \\
Myogenin & - & + & - & + \\
CK (Pan) & - & + & - & $+/-$ \\
EMA & - & + & - \\
CEA & - & + & - \\
\hline
\end{tabular}

+, positivity; +/-, rare positivity. CEA, carcinoembryonic antigen; CK, cytokeratin; EMA, epithelial membrane antigen; eMPNST, epithelioid malignant peripheral nerve sheath tumor; P; MTT, malignant triton tumor.

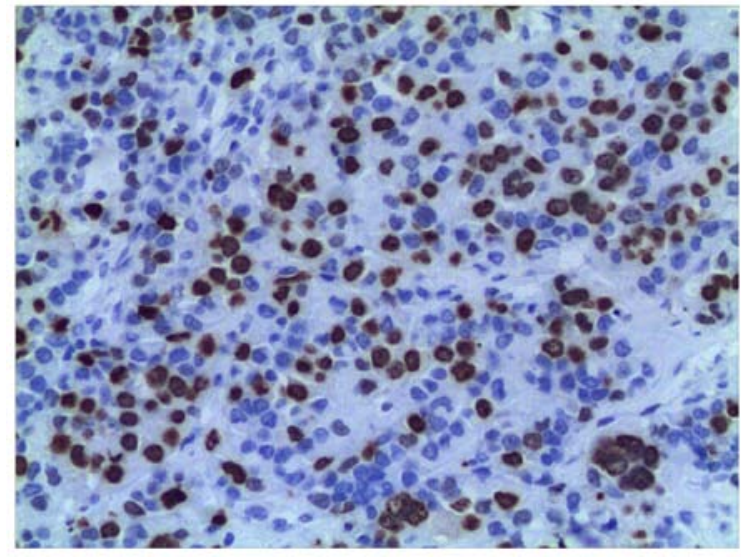

Figure 5. Ki-67 proliferation index staining of $\sim 40 \%$.

has not been detected in the patient in the 4 years since the initial operation in 2014. However, the recovery of the patient continues to be evaluated.

\section{Discussion}

The eMPNST is a distinct subtype of MPNST that accounts for $<5 \%$ of all cases. MPNST commonly affects patients aged between 40 and 70 years old, with no sex predilection (16). eMPNSTs primarily involve the trunk and lower extremities of the body, and are more superficial than MPNSTs (8). The association between eMPNST and NF-1 is rare and occurs in $<2 \%$ of all MPNSTs (9). To the best of our knowledge, only 5 cases in the English literature describing eMPNST associated with NF-1 have been reported $(6,13,15,17,18)$. A characteristic histological feature of eMPNST is the predominance $(\geq 50 \%)$ or prevalence of epithelioid tumor cells arranged in multinodular growth pattern, with myxoid and/or fibrous stroma, and usually admixed with a diverse proportion of spindle cell components $(8,13,14)$. Tumor cells surrounded by hyalinized or myxoid stroma frequently grow in cords, strands, nests or sheets (8). Polygonal epithelioid cells have characteristic round or oval-shaped nuclei, vesicular chromatin, variably prominent basophilic nucleoli and an abundant 
eosinophilic or amphophilic cytoplasm, with well-defined cell membranes (19). The nuclear atypia is usually moderate to severe and widespread, and the mitotic activity that reflects the degree of nuclear atypia is consistently present, with a mean number of 7/10 HPF (13). The immunohistochemical characteristics of eMPNSTs usually exhibit diffuse positivity for S-100 proteins and vimentin, whereas focal and patchy positive S-100 staining is rare $(8,13,20)$. The SMARCB1 (INI1/BAF47/SNF5) gene is located on the chromosome 22 (22q11.2) and functions as a tumor suppressor gene, deletions and/or mutations of SMARCB1 gene resulting in the loss of nuclear integrase interactor 1 (INI1) protein expression have been detected in eMPNST (14). A previous study reported that eMPNSTs exhibit a loss of expression of INI1 ranging from 50-67\% (14). Negative staining for second-line melanoma markers, including HMB45, melan A and MIFT, is crucial for distinguishing eMPNSTs from melanoma. Notably, focal positive staining for melan A and HMB45, and negative staining for S-100 in eMPNST cases have also been reported $(13,19)$. In addition, eMPNST is rarely associated with NF-1, and occurs in a variety of anatomical locations. Subsequently, a precise diagnosis of eMPNST can be difficult. The main differential diagnosis of eMPNST comprises other skin tumors with an epithelioid morphology, including malignant melanoma, metastatic poorly differentiated carcinoma, epithelioid sarcoma and myoepithelial carcinoma (8). In addition, distinction from other differentiations of MPNST, including glandular MPNST and rhabdomyoblastic MPNST, is also critical. Malignant melanoma or metastatic melanoma may be characterized histologically by an epithelioid phenotype, and often exhibits diffuse positivity for S-100 protein, which makes it harder to distinguish from eMPNST $(13,14)$. However, melanoma-associated markers, including melan A, HMB45, tyrosinase and MITF, are generally absent in eMPNST, in contrast with melanoma of epithelioid morphology (21). In particular, MITF and S-100 positive staining, which, to the best of our knowledge, has not yet been detected in eMPNSTs, is therefore considered to be highly specific for melanoma (21). In addition, malignant melanoma commonly exhibits expression of INI1, whereas the loss of INI1 nuclear expression is observed in 50-67\% of eMPNST cases (20). Observation of the tumor ultrastructure by electron microscope can provide a solution to the difficulties associated with differential diagnosis, since the presence of atypical junctional components or melanin pigmentation can be indicative of melanoma (13). Careful examination to exclude the history of melanoma or the clinical presence of melanoma is also crucial in order to differentiate eMPNST from malignant melanoma in the majority of cases. Epithelioid sarcoma can be differentiated from eMPNST through immunohistochemical analysis. For example, epithelioid sarcoma is positive for cytokeratin and negative for S-100, and exhibits a loss in INI1 expression (8). In addition, CD34 is positive in $~ 50 \%$ of epithelioid sarcomas $(8,13)$. Negative staining results for cytokeratins can serve in distinguishing eMPNSTs from metastatic poorly differentiated carcinoma, with a lack of staining or focal positivity in the former, and positivity observed in the latter (20). Myoepithelial carcinoma shares similar morphological characteristics with eMPNST, and is predominantly comprised of epithelioid cells. However, unlike eMPNST, myoepithelial carcinoma is positive for multiple cytokeratins, including CK-AE1/AE3, CAM 5.2 and/or EMA (18). Notably, myoepithelial carcinoma exhibits unusually strong and multifocal S-100 positivity and a loss of INI1 expression of up to $40 \%$ (8). Malignant triton tumor (MTT) is a variant of MPNST with rhabdomyoblastic differentiation $(22,23)$. The immunohistochemical characteristics of MTT overlap with those of eMPNST; however, it can be distinguished from eMPNST by the desmin, myo-D1 and myogenin positivity of rhabdomyoblasts in MMT, and consistent negativity in eMPNST (23). Glandular MPNST is an MPNST subset with glandular differentiation. Histologically, the majority of glandular PNSTs are composed of a malignant spindle cell component and a benign glandular component (24). Unlike eMPNST, glands stain positive for epithelial markers, including keratins and epithelial membrane antigen, and neuroendocrine markers, including somatostatin, chromogranin, calcitonin and Leu-7 $(23,24)$. Immunohistochemistry is therefore a useful technique to differentiate eMPNSTs from other differential diagnoses of MPNST. The useful immunohistochemical features of differential diagnosis are summarized in Tables I and II. The patient from the present study exhibited clinical characteristics of NF-1, and the histology and immunophenotype were consistent with an eMPNST diagnosis.

MPNSTs are commonly considered to be aggressive malignant soft-tissue tumors. The primary treatment for MPNST is a complete surgical resection with negative margins, and the surgical margin status is a crucial prognostic factor for MPNST (3). Wong et al (25) reported that the 5-year survival rates of patients with MPNST who received surgical treatment with and without negative margins were 67 and 22\%, respectively. However, complete resection with negative margins cannot always be achieved due to unacceptable complications, including severe loss of vascular function. Gachiani et al (26) reported that amongst 34 patients with MPNST who received surgical tumor resection over a period of 40 years, successful complete excision only occurred in 16 patients (47\%). Previous studies have recommended the use of postoperative radiation therapy to treat MPNST in order to decrease the incidence of local recurrences, especially in tumors that exhibit the following characteristics: Lesions $>5 \mathrm{~cm}$ in size, high tumor grade, unresectable lesions and positive margins (25,27). Basso-Ricci (27) reported that among 25 patients with MPNST who were followed up for $>3$ years, 14 were free of disease following combined radiological-surgical treatment, representing a rate that was higher than that found in the other patient cohort who underwent surgery only. Wong et al (25) reported that patients who received irradiation compared with patients who did not had 5-year overall survival rates of 72 and $50 \%$, respectively. However, the risk-benefit profile of adjuvant radiation therapy must be carefully discussed with all patients, as it increases the risk of radiation-induced sarcomas (4). MPNSTs are typically considered to be insensitive to chemotherapy. The role of chemotherapy in MPNSTs is commonly limited to the management of metastatic MPNST in patients with tumors that cannot be resected (28). The outcome of MPNST treatment remains poor, with a reported local recurrence rate between 40-65\% and an overall 5-year survival rate $<50 \%(3,12)$. As a subset of MPNSTs; however, the biological features and prognosis in patients with eMPNST remain unclear due to the small patient cohorts, the lack of large-scale clinical research and the 


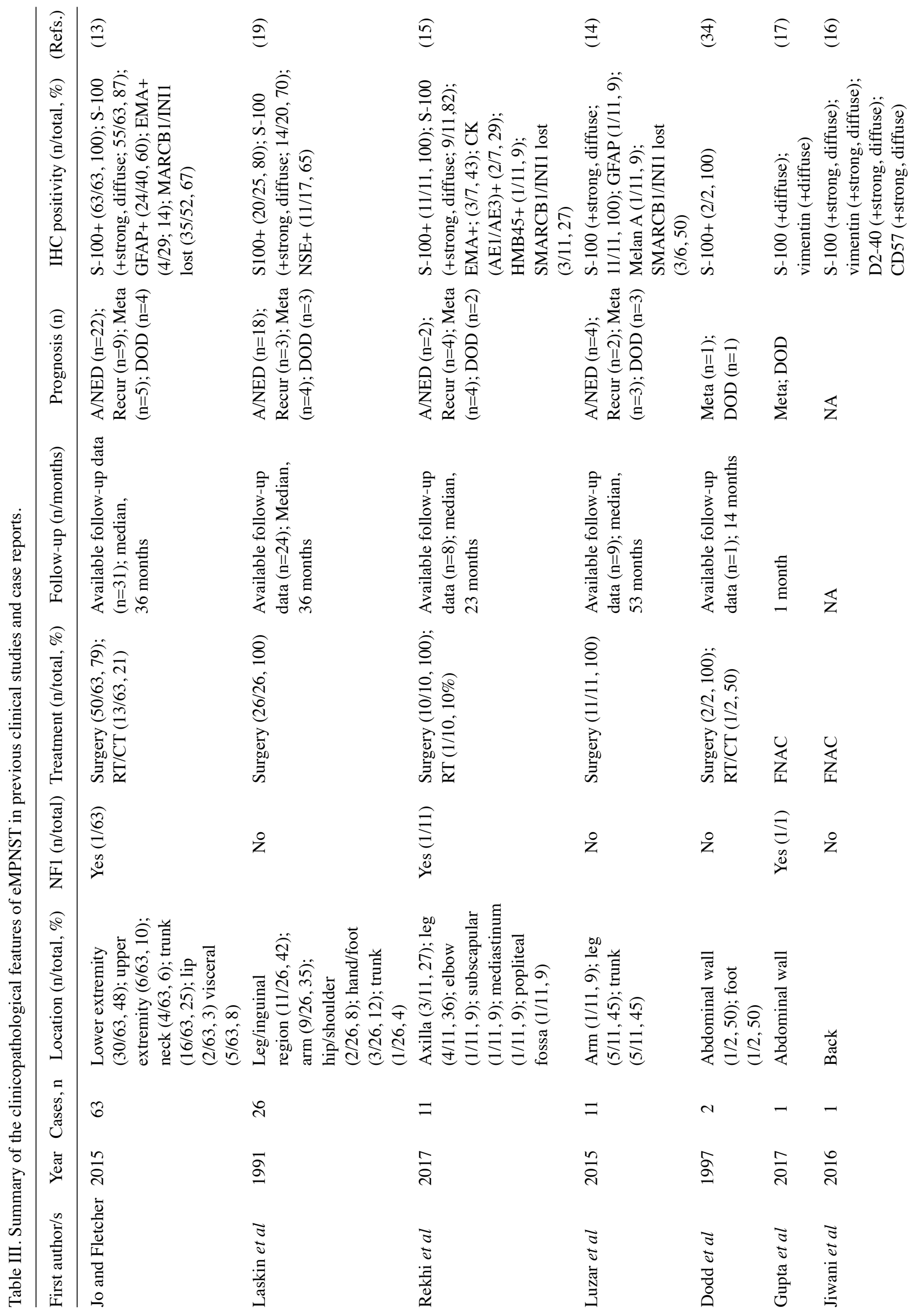




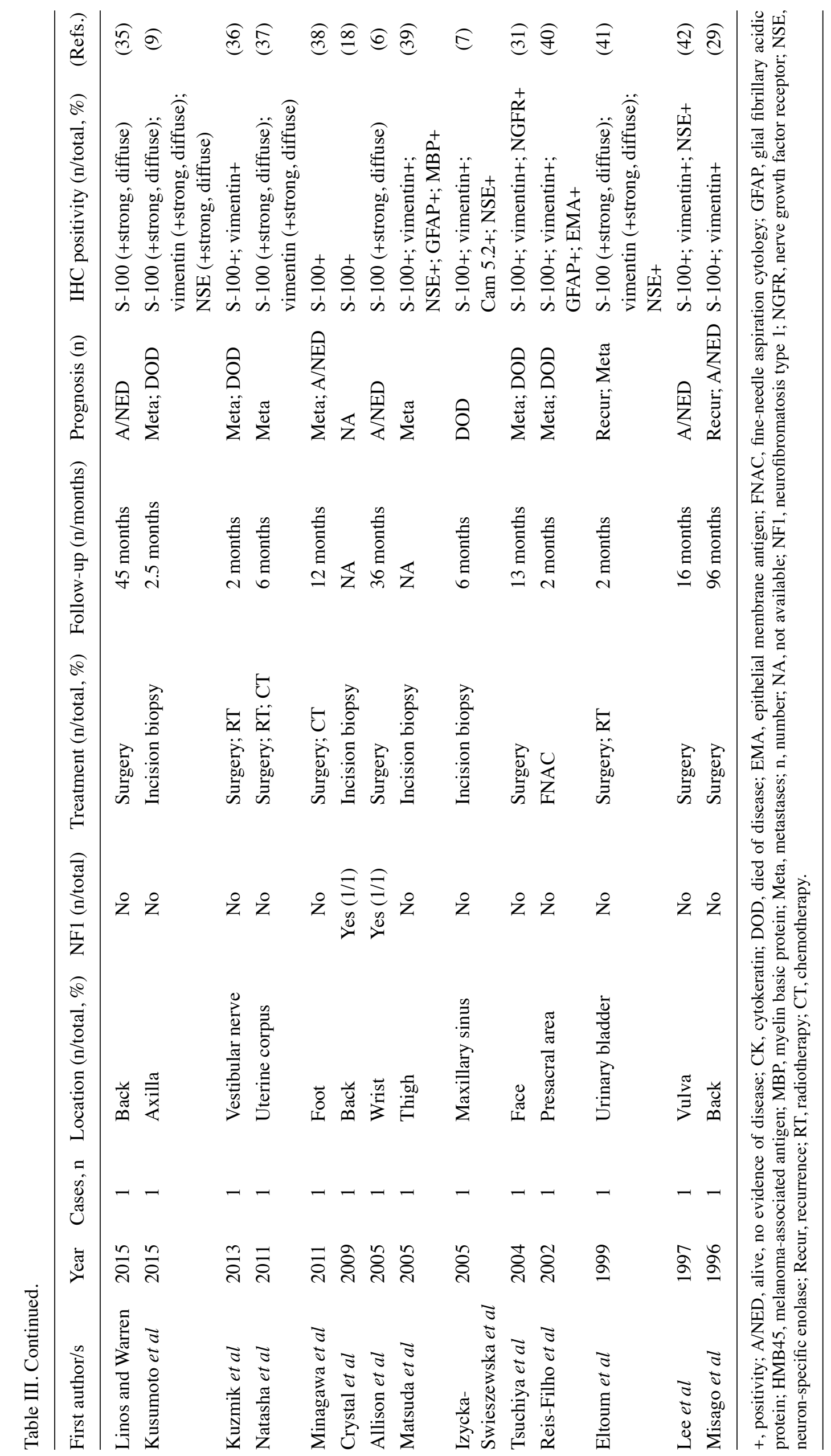


variation in survival rates. The clinicopathological characteristics of eMPNST from the aforementioned clinical studies and case reports are summarized in Table III. The largest study, including 63 cases, was presented by Jo and Fletcher (13). The study reported that follow-up data were available for 31 cases over a median duration of 26 months, and that 9 patients developed local recurrence and 4 patients succumbed to metastatic disease. It was concluded that there is a lower risk for recurrence, metastasis and disease-associated mortality in patients with eMPNST compared with that in patients with conventional MPNST. The study by Laskin et al (19) analyzed 26 patients with eMPNSTs, including 16 patients $(61.5 \%)$ with tumors in superficial locations and 10 patients $(38.5 \%)$ with tumors in deep-seated locations. The results revealed that eMPNSTs in superficial locations are less aggressive than the deep-seated tumors. The patient included in the present study experienced two recurrences, one at 6 months after the initial surgery, and the other one at 5 months after the second surgery. Postoperative follow-up at 2 years revealed no evidence of recurrent disease following the final surgery, which may be due to the use of a wide resection combined with radiotherapy at a dose of $60 \mathrm{~Gy}$. Furthermore, metastasis has not occurred in the patient in the 4-year period following initial surgery. In the present study, the behavior of the superficial eMPNST was favorable, despite repeated recurrences. Similarly, Misago et al (29) reported that in a patient with superficial eMPNST located on the back, who underwent three operations and had relapses following two previous surgeries, no evidence of recurrence was observed at $\sim 2$ years following final surgery. Despite repeated recurrences, the patient did not exhibit any signs of metastasis for $\sim 8$ years following the first operation. Superficial eMPNST is generally considered to have benign clinical behavior (13), which may be due to its early clinical manifestation and detection of recurrence, the superficial location and the fact that it can be clinically treated at an early stage by radical excision with free margins. In addition, other sarcomas, including superficial/cutaneous leiomyosarcoma, have a more favorable prognosis than their deep-seated counterparts (30). However, previous studies and case reports on eMPNSTs suggested that superficial eMPNSTs may not have such favorable clinical progression. For example, in the study by Rekhi et al (15), data from the follow-up performed on 8 patients with superficial eMPNST over a median duration of 26 months revealed that tumors exhibit the same potentially aggressive biological behaviors regardless of tumor depth. Although most patients in the study had undergone surgical resection, $>50 \%$ of surgical margin statuses were either positive or unclear, which could affect the accuracy of the conclusion. Tsuchiya et al (31) reported that a patient with superficial eMPNST on the face, who had undergone three surgical excisions and three metastases during a period of 12 months, succumbed to multiple metastases to the lung, liver and brain 13 months after the initial surgery. In the present study, lesions were present on the cheek near the corner of the eye, and the difficulty in completely excising the tumor, and the skin defects which remained following surgical removal of the tumor required a skin graft from other areas of the body each time. These reasons may have resulted in the tumor not being removed completely with an adequate surgical margin. The primary treatment for MPNST consisted of urgent radical excision with a free margin. However, it is technically challenging to obtain a free margin resection, due to the large size of the tumor or the difficulty in accessing the anatomical location of the tumor, including the head and neck area. This may explain the poor prognosis of MPNSTs of the head and neck $(32,33)$. Although superficial eMPNSTs may carry a good prognosis, they have the potential for recurrence and metastasis, and long-term follow-up for every patient is therefore essential.

In conclusion, eMPNST is histologically characterized by the predominance of epithelioid tumor cells, with diffuse positivity for S-100 protein, and is rarely associated with NF-1. eMPNST is a diagnostically challenging neoplasm with an extensive differential diagnosis. Appropriate clinical and histological evaluation with a suitable immunohistochemical panel is crucial to determine the correct diagnosis of this rare tumor. The principal treatment for eMPNST consists of a complete surgical resection with negative margins; however, subsequent radiotherapy may be crucial to reduce the risk of local recurrences. eMPNSTs are frequently identified in superficial locations and this location confers a good prognosis. However, eMPNSTs may have the potential for recurrence and metastasis. Long-term patient follow-up is therefore crucial.

\section{Acknowledgements}

The authors would like to thank Dr YeYuan Chen (Department of Radiology, The First Affiliated Hospital of Nanchang University) for the imaging data and description.

\section{Funding}

The present study was supported by The Project Fund of Technology Department of Jiangxi Province, China (grant no. 20141040).

\section{Availability of data and materials}

All data generated or analyzed during this study are included in this published article.

\section{Authors' contributions}

PD, JZ, CH, MYY, YXL, JSZ and QHT participated in the treatment of this patient and helped to draft the manuscript. ZDZ performed the pathological examination and immunohistochemical staining. PD and JZ performed the data analyses and wrote the manuscript. JSZ and QHT gave final approval of the version to be submitted. All authors read and approved the final manuscript.

\section{Ethics approval and consent to participate}

The present study was approved by the Ethics Review Committee of The First Affiliated Hospital of Nanchang University (Nanchang, China).

\section{Patients consent for publication}

Written informed consent was provided by the patients. 


\section{Competing interests}

The authors declare that they have no competing interests.

\section{References}

1. Tonsgard JH: Clinical manifestations and management of neurofibromatosis type 1. Semin Pediatr Neurol 13: 2-7, 2006.

2. Ferner RE and Gutmann DH: Neurofibromatosis type 1 (NF1): Diagnosis and management. Handb Clin Neurol 115: 939-955, 2013.

3. James AW, Shurell E, Singh A, Dry SM and Eilber FC: Malignant peripheral nerve sheath tumor. Surg Oncol Clin N Am 25: 789-802, 2016

4. Farid M, Demicco EG, Garcia R, Ahn L, Merola PR, Cioffi A and Maki RG: Malignant peripheral nerve sheath tumors Oncologist 19: 193-201, 2014.

5. Kahn J, Gillespie A, Ondos J, Dombi E, Camphausen K, Widemann BC and Kaushal A: Radiation therapy in management of sporadic and neurofibromatosis type 1-associated malignant peripheral nerve sheath tumors. Front Oncol 4: 324, 2014.

6. Allison KH, Patel RM, Goldblum JR and Rubin BP: Superficial malignant peripheral nerve sheath tumor: A rare and challenging diagnosis. Am J Clin Patho 124: 685-692, 2005.

7. Izycka-Swieszewska E, Drogoszewska B, Filipowicz J, Szurowska E, Kaminski M and Jaskiewicz K: Epithelioid malignant peripheral nerve sheath tumor involving maxillary sinus. Neuropathology 25: 341-345, 2005.

8. Luzar B and Falconieri G: Cutaneous malignant peripheral nerve sheath tumor. Surg Pathol Clin 10: 337-343, 2017.

9. Kusumoto E, Yamaguchi S, Sugiyama M, Ota M, Tsutsumi N, Kimura Y, Sakaguchi Y, Kusumoto T, Ikejiri K, Nakayama Y and Momosaki S: Huge epithelioid malignant peripheral nerve sheath tumor in the left axilla: A case report. Surg Case Rep 1: 64, 2015

10. Thway K: Malignant peripheral nerve sheath tumor: Pathology and genetics. Ann Diagn Pathol 18: 109-116, 2014.

11. Graefe C, Eichhorn L, Wurst P, Kleiner J, Heine A, Panetas I, Abdulla Z, Hoeft A, Frede S, Kurts C, et al: Optimized Ki-67 staining in murine cells: A tool to determine cell proliferation. Mol Biol Rep: May 15, 2019 (Epub ahead of print). doi: 10.1007/s11033019-04851-2.

12. Hagi T, Nakamura T, Yokoji A, Matsumine A and Sudo A: Medullary metastasis of a malignant peripheral nerve sheath tumor: A case report. Oncol Lett 12: 1906-1908, 2016.

13. Jo VY and Fletcher CD: Epithelioid malignant peripheral nerve sheath tumor: Clinicopathologic analysis of 63 cases. Am J Surg Pathol 39: 673-682, 2015.

14. Luzar B, Shanesmith R, Ramakrishnan R, Fisher C and Calonje E: Cutaneous epithelioid malignant peripheral nerve sheath tumour: A clinicopathological analysis of 11 cases. Histopathology 68: 286-296, 2015

15. Rekhi B, Kosemehmetoglu K, Tezel GG and Dervisoglu S: Clinicopathologic features and immunohistochemical spectrum of 11 cases of epithelioid malignant peripheral nerve sheath tumors, including INI1/SMARCB1 results and BRAF V600E analysis. Apmis 125: 679-689, 2017.

16. Jiwani S, Gokden M, Lindberg M, Ali S and Jeffus S: Fine-needle aspiration cytology of epithelioid malignant peripheral nerve sheath tumor: A case report and review of the literature. Diagn Cytopathol 44: 226-231, 2016.

17. Gupta RK, Saran RK, Ghuliani D, Garg L and Das A: Metastatic epithelioid malignant peripheral nerve sheath tumor in a known case of neurofibromatosis-1, cytomorphological appearance, and critical analysis of immunohistochemistry. Indian J Med Paediatr Oncol 38: 387-390, 2017

18. Crystal T, Najwa S, Owen LG, Malone JC and Billings SD: Cutaneous malignant peripheral nerve sheath tumors. J Cutan Pathol 36: 896-900, 2009.

19. Laskin WB, Weiss SW and Bratthauer GL: Epithelioid variant of malignant peripheral nerve sheath tumor (malignant epithelioid schwannoma). Am J Surg Pathol 15: 1136-1145, 1991.

20. Hornick JL, Paola DC and Fletcher CD: Loss of INI1 expression is characteristic of both conventional and proximal-type epithelioid sarcoma. Am J Surg Pathol 33: 542-550, 2009.

21. Miettinen M, Fernandez M, Franssila K, Gatalica Z, Lasota J and Sarlomo-Rikala M: Microphthalmia transcription factor in the immunohistochemical diagnosis of metastatic melanoma: Comparison with four other melanoma markers. Am J Surg Pathol 25: 205-211, 2001.
22. Li G, Liu C, Liu Y, Xu F, Su Z, Wang Y, Ren S, Deng T, Huang D, Tian Y and Qiu Y: Analysis of clinical features and prognosis of malignant triton tumor: A report of two cases and literature review. Oncol Lett 10: 3551-3556, 2015.

23. Khin T and Cyril F: Malignant peripheral nerve sheath tumor: Pathology and genetics. Ann Diagn Pathol 18: 109-116, 2014.

24. Nagasaka T, Lai R, Sone M, Nakashima T and Nakashima N: Glandular malignant peripheral nerve sheath tumor: An unusual case showing histologically malignant glands. Arch Pathol Lab Med 124: 1364-1368, 2000.

25. Wong WW, Hirose T, Scheithauer BW, Schild SE and Gunderson LL: Malignant peripheral nerve sheath tumor: Analysis of treatment outcome. Int J Radiat Oncol Biol Phys 42: 351-360, 1998

26. Gachiani J, Kim D, Nelson A and Kline D: Surgical management of malignant peripheral nerve sheath tumors. Neurosurg Focus 22: E13, 2007.

27. Basso-Ricci S: Therapy of malignant schwannomas: Usefulness of an integrated radiologic. Surgical therapy. J Neurosurg Sci 33: 253-257, 1989.

28. Ferner RE and Gutmann DH: International consensus statement on malignant peripheral nerve sheath tumors in neurofibromatosis. Cancer Res 62: 1573-1577, 2002.

29. Misago N, Ishii Y and Kohda H: Malignant peripheral nerve sheath tumor of the skin: A superficial form of this tumor. J Cutan Pathol 23: 182-188, 1996.

30. Fields JP and Helwig EB: Leiomyosarcoma of the skin and subcutaneous tissue. Cancer 47: 156, 1981.

31. Tsuchiya D, Takamura H, Saito K, Kashiwa H, Maeda K and Yamashita H: Immunohistochemical diagnosis of a rare case of epithelioid malignant peripheral nerve sheath tumor with multiple metastases. Jpn J Ophthalmol 48: 565-569, 2004.

32. Harald V, Noriaki N, Eva W, Wenzel J, Bieber T, Wendtner CM, Reinhard G and Schmid-Wendtner MH: Malignant peripheral nerve sheath tumor of the scalp: Case report and review of the literature. Dermatol Surg 37: 1684-1688, 2011.

33. Hanai U, Akamatsu T, Kobayashi M, Tsunoda Y, Hirabayashi K, Baba T, Atsumi H and Matsumae M: A case of occipital malignant peripheral nerve sheath tumor with neurofibromatosis type 1. Tokai J Exp Clin Med 41: 130-134, 2016.

34. Dodd LG, Scully S and Layfield LJ: Fine-needle aspiration of epithelioid malignant peripheral nerve sheath tumor (epithelioid malignant schwannoma). Diagn Cytopathol 17: 200-204, 1997.

35. Linos K and Warren S: A misdiagnosed melanoma: A case of cutaneous epithelioid malignant peripheral nerve sheath tumor. Dermatol Online J 21: 13030/qt319176xb, 2015.

36. Kuzmik GA, Michaelides EM, Chiang VL, Nonaka Y, Fukushima T, Vortmeyer AO and Bulsara KR: Rapidly progressive epithelioid malignant peripheral nerve sheath tumor of the vestibular nerve. Otol Neurotol 34: 1739, 2013.

37. Natasha G, Bharat R, Pallavi S and Jambhekar NA: Epithelioid malignant peripheral nerve sheath tumor of the uterine corpus. Ann Diagn Pathol 15: 441-445, 2011.

38. Minagawa T, Shioya R, Sato C, Shichinohe R, Yasui G, Ishikawa $\mathrm{K}$ and Takahashi $\mathrm{H}$ : Advanced epithelioid malignant peripheral nerve sheath tumor showing complete response to combined surgery and chemotherapy: A case report. Case Rep Oncol Med 2011: 705345, 2011.

39. Matsuda Y, Saoo K, Hosokawa K, Yamakawa K, Yokohira M, Zeng Y, Takeuchi H, Iwai J, Shirai T, Obika K and Imaida K: Epithelioid malignant peripheral nerve sheath tumor. Report of a case with inflammatory infiltration. Pathol Res Pract 201: 355-360, 2005

40. Reis-Filho JS, Pope LZ, Balderrama CM, Fillus-Neto J and Schmitt FC: Epithelioid malignant peripheral nerve sheath tumour: Case report and review of the previously published cases. Cytopathology 13: 54-63, 2002.

41. Eltoum IA, Moore RJ, Cook W, Crowe DR, Rodgers WH and Siegal GP: Epithelioid variant of malignant peripheral nerve sheath tumor (malignant schwannoma) of the urinary bladder. Ann Diagn Pathol 3: 304, 1999.

42. Lee YS, Choi YJ, Kang CS, Kang SJ, Kim BK and Shim SI: Purely epithelioid malignant peripheral nerve sheath tumor of the vulva. J Korean Med Sci 12: 78-81, 1997.

This work is licensed under a Creative Commons Attribution-NonCommercial-NoDerivatives 4.0 International (CC BY-NC-ND 4.0) License. 